\title{
Aphalia: An Extremely Rare Congenital Genitourinary Malformation-A Case Report
}

\author{
Melkamu Berhane $^{1 *}$, Gersam Abera ${ }^{2}$, Seifu Alemu ${ }^{2}$, Beza Eshetu ${ }^{1}$
}

\footnotetext{
OPEN ACCESS

Citation: MelkamuBerhane, Gersam Abera, Seifu Alemu, BezaEshetu. Aphalia: An Extremely Rare Congenital Genitourinary Malformation - A Case Report. Ethiop J Health Sci. 2019;29(3):409.

doi:http://dx.doi.org/10.4314/ ejhs.v29i3.13 Received: October26, 2018

Accepted:April 4, 2019

Published: May 1, 2019

Copyright: (C) MelkamuBerhane, et al. This is an open access article distributed under the terms of the Creative Commons Attribution License, which permits unrestricted use, distribution, and reproduction in any medium, provided the original author and source are credited.

Funding:Nil

Competing Interests: The authors declare that this manuscript was approved by all authors in its form and that no competing interest exists.

Affiliation and Correspondence:

${ }^{1}$ Department of Pediatric and Child

Health, Jimma University

${ }^{2}$ Department of Surgery, Jimma University

*Email:melkamuberhane@yahoo.c om
}

\section{ABSTRACT}

BACKGROUND: Congenital aphalia is an extremely rare, urogenital malformation of the external genitalia characterized by congenitally absent penis in a karotypically $X Y$ child. It occurs 1 in 30 million live births.So far, only less than 100 cases have been reported.

CASE DETAILS: A 3-hour old newborn was diagnosed with aphalia after being referred from health center for respiratory distress and congenital malformation of the external genitalia. He had deranged renal function tests, hypoplastic kidneys, small patent foramen ovale and ventricular septal defect. Management of the acute conditions and parental counseling were done, but he passed away on the $8^{\text {th }}$ day of life, due to the underlying diseases.

CONCLUSION: A patient with aphalia needs thorough evaluation for possible associated malformations. Management is multistaged and multidisciplinary. Parental counselling should be started as early as possible, and their decision should be respected.

KEYWORDS: Aphalia, neonate, malformations, gender reassignment

\section{INTRODUCTION}

Aphalia, congenitally absent penis, is thought to result from the nonformation or failure of development of the fetal genital tubercle or failure in caudal migration of the urogenital sinus between the third and sixth weeks of embryonic development(1-4). The development of the penis depends on dihydrotestesterone from the testes, to which the genital tubercle is sensitive. By 9weeks of gestation, the genital tubercle starts to lengthen under the influence of testosterone and becomes either the penis or the clitoris(4-5). The level of testosterone after 8 weeks of gestation determines phallus size at birth.

The presentation is usually in the neonatal period but may be delayed for months or years(3). Clinically, the penis is absent, the scrotum is well developed, and the testes are usually well developed and descended. The urethra usually opens adjacent to the anus, and in some cases, into the rectum(1-3,5).

The management of aphalia is challenging both to the parents and the professionals due to the associated urological and psychological consequences. It requires multistage and 
multidisciplinary approach including pediatricians, pediatric surgeons, geneticists, endocrinologists and psychologists. Immediate management includes prevention of infra-vesical obstructive uropathy and treatment of life threatening associated malformations. In the second stage, meatal opening may be advanced near the pubic region(3-5).

Gender assignment should be done in the neonatal period, otherwise should be individualized based on age of presentation, sex of rearing, psychological status of the child and opinion of the family. Thorough discussion should be made with the parents before any attempt of gender reassignment, surgical intervention is made, and their decision should be respected. The discussions should take into consideration the future socio-sexual aspect, fertility and gender of the child(3-5). We report a case of a 3-hour old newborn presented to a neonatal intensive care unit.

\section{CASE REPORT}

A 3-hour old newborn was born to a 21years old para I mother after amenorrhea of 9 months. The mother had antenatal care follow-up, and had no problem identified during the pregnancy.There was no other significant history. The duration of labor was 12 hours and rupture of membrane was immediately before delivery. The baby did notcry immediately after birth, was resuscitated for 10minutes, and referred to our hospital for respiratory distress.
On arrival, he was acutely sick looking and in respiratory distress. Apical heart rate, respiratory rateand temperature were $124^{\prime}, 82^{\prime}$ and $34.9^{\circ} \mathrm{C}$ respectively. Weight andhead circumference were $2300 \mathrm{gm}$ and $35 \mathrm{~cm}$ respectivelywhereas gestational age was 34-36 weeks. Respiratory examination revealed inter-costal and sub-costal retractions, flaring of ala nasae and coarse creptations bilaterally. The penis was notvisible or palpable; the scrotum was well developed and pendulous with bilaterally palpable testes (Figure 1). The anus was patent with a urethral opening on the anterior aspect of the anal canal just at the anal orifice.

Laboratory investigations showed normal haematocrit and random blood sugar level but elevated serum creatinine $(4.93 \mathrm{mg} / \mathrm{dl})$ and blood urea nitrogen $(78.5 \mathrm{mg} / \mathrm{dl})$. We did not have repeat measurements. Abdominal ultrasound and echocardiography revealed bilateral hypoplastic kidneys and small patent foramen ovale and ventricular septal defect (VSD) respectively; other internal organs were normal.

He was initially managed for prematurity, perinatal asphyxia, and respiratory distress syndrome while being evaluated by pediatric surgery fellows. Then, the parents were counseled about the condition and possible subsequent management. However, the neonate passed away of the underlying medical conditions on the $8^{\text {th }}$ day of life before any further investigation or management was made. Although it was important to establish the cause of death, autopsy was not done. 


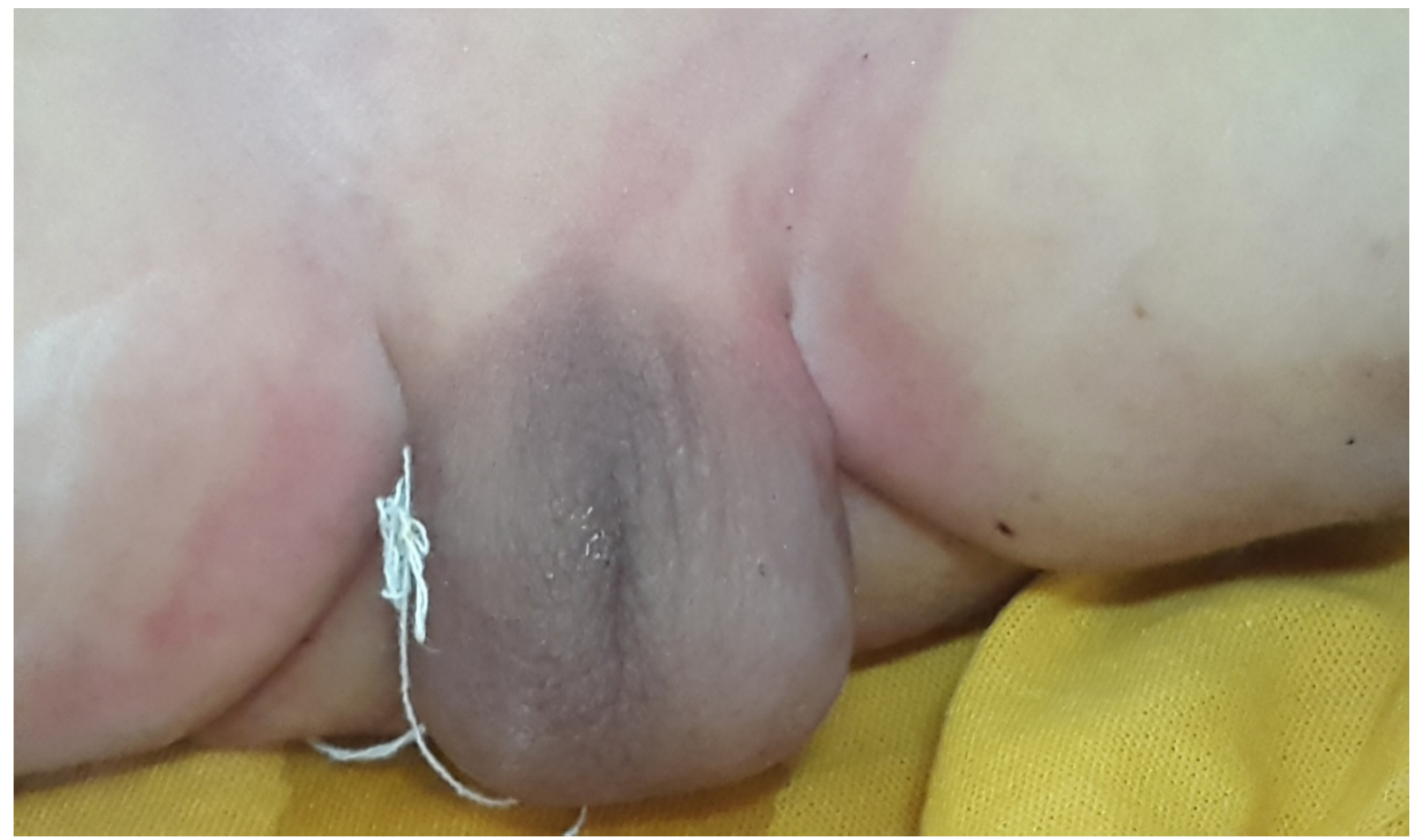

Figure 1: A 3-day old neonate with Aphalia

\section{DISCUSSION}

Aphalia should be differentiated from other malformations of the genitourinary system like severe hypospadias, epispadias, concealed penis, micropenis, intrauterine penile amputation and disorders of sexual differentiation(4). Evaluation of the patient with aphalia should include genetic evaluation and evaluation for associated malformations. Common associated malformations include genitourinary anomalies (renal agenesis, hypoplastic kidneys, hydronephrosis, vesicourethral reflux, hypoplastic bladder, vesicocolic fistula, and agenesis of the prostate and seminal vesicles) and gastrointestinal malformations (anteriorly placed anus, tracheoesophageal fistula, and annular pancreas). Other associated malformations include pigeon chest, hemivertebrae, club feet, shortened forearms, accessory earlobes and low set ears (4-5). Our patient had deranged renal function tests, bilateral hypoplastic kidneys and VSD, but we did not do genetic and other tests because of the unavailability of the tests and the early death of the infant.
One of the most important ways of classification of aphalia was proposed by Skoog and Belman in 1984. In post-sphincteric $(60 \%)$, the urethra opens near the anterior anal verge or any point on the perineum in midline, over the pubis or anterior scrotum.In pre-sphincteric $(28 \%)$, there is prostatorectal (urethra-rectal) fistula whereas in the less frequent type, there is urethral atresia with vesico-rectal fistula. The more proximal the urethral opening is, the higher the likelihood of having sever forms of aphalia, associated malformations and mortality (4). Our patient had post-sphincteric type.

The previous recommendation was female gender reassignment with urethral perineal transposition, early bilateral orchiectomy and feminizing genitoplasty in the neonatal period or at the time of presentation. Vaginoplasty is undertaken at the time of puberty, followed by estrogen therapy for the development of female secondary sexual characteristics $(1,2)$. In our case, the baby passed away before the parents decided on gender assignment.

The current recommendation is towards male gender reassignment with reconstruction of a normally functioning penis which is 
aesthetically acceptable, has penetrating capacity, provides tactile and erogenous sensation, adequate reproductive function and the ability to urinate standing. The justifications for the current recommendation were the drawbacks associated with the female gender reassignment (lack of menstruation, infertility, development of male typical shift and lifelong steroids use(2,5). Many of the patients with aphalia die during the first few weeks of life because of the multiple associated malformations $(1,2)$ which was also the case in our patient.

In conclusion, aphalia is an extremely rare malformation which might be associated with malformations in multiple other organ systems. It needs thorough evaluation as well as multistage and multidisciplinary treatment. Parental counseling and decision playsa critical role in the management of a child with aphalia.

\section{ACKNOWLEDGEMENT}

We would like to thank the care takers for giving us their consent.

\section{REFERENCES}

1. Aslanabadi S, Zarrintan S, Abdollahi H, Rikhtegar R, Beheshtirouy S, Bade D. A Rare Case of Aphallia with Right Kidney Hypoplasia and Left Kidney Dysplasia. Arch Iran Med. 2015; 18(4):4:257-259.

2. Shamsa A, Kajbafzadeh AM, Parizadeh SMJ, Zare MA, Abolbashari M. Aphallia associated with Urethro-Rectal Fistula and Stones in the Bladder and Urethra. Saudi J Kidney Transpl. 2008;19(3):435-438.

3. Wang H, Guo K, Wang J, Liu L, Li F. Aphallia in an adult male with 46,XY karyotype. Int $J$ Urol. 2011;18:540-542.

4. Bangroo A.K, Ramji Khetri, Shashi Tiwari. Penile Agenesis. J Indian Assoc Pediatr Surg. 2005;10(4):256-258.

5. Shastri S, Sharma D, Kumar R. A case report of aphallia with urorectal septum malformation sequence in a newborn: a very rarely seen condition. Int Med Case Rep J. 2015;8:317-320. 